Supporting Information

\title{
Metal-labeled Aptamers as Novel nanoprobes for Imaging Mass Cytometry Analysis
}

Youyi Yuł, Jingqi Dangł, Xiao Liu, Liping Wang, shanhe Li, Ting Zhang, and

Xianting Ding*

Institute for Personalized Medicine, School of Biomedical Engineering, Shanghai Jiao

Tong University, Shanghai, 200030, China.

\section{Supporting figures}

Table S1 The yield of synthesized probes

Figure S1 The UV spectra of synthesized probes

Figure S2 The mass spectrum of aptamer and metal-labeled aptamer

Figure $\mathbf{S 3}$ The HE and IHC of the prostate cancer tissues sections

Figure S4 The IMC images stained with probes in series of concentration

Figure S5 The percentage of PSMA stained by ${ }^{167}$ Er-YPSMA-1

Figure S6 The percentage of PSMA stained by ${ }^{167} \mathrm{Er}-\mathrm{A} 10-3.2$

Figure S7 Scheme describing hypothesis that the aptamer could access more epitopes

Figure S8 The IMC image of blocking experiment

Figure S9 The IMC images stained with two metal-tagged antibodies

Figure S10 The percentages of PSMA, PSA stained by ${ }^{167}$ Er-A10-3.2, ${ }^{176} \mathrm{Yb}-\Delta \mathrm{Psap} 4 \# 5$.

Figure S11 The percentages of PSMA, PSA stained by ${ }^{167}$ Er-YPSMA-1, ${ }^{176} \mathrm{Yb}$ EP1588Y 
Table S1 The yield experimental data of the synthesized probes

\begin{tabular}{ccccc}
\hline Mass tag & $\mathbf{m}_{\mathbf{0}}(\boldsymbol{\mu g})$ & $\mathrm{C}_{\mathbf{x}}(\mathrm{ng} / \boldsymbol{\mu L})$ & $\mathrm{V}(\boldsymbol{\mu L})$ & yield \\
\hline${ }^{167} \mathbf{E r}-\mathbf{A 1 0 - 3 . 2}$ & 6.60 & 53.17 & 100 & $80.6 \%$ \\
${ }^{167}$ Er-YPSMA-1 & 50 & 246.0 & 100 & $49.2 \%$ \\
${ }^{\mathbf{1 7 6}} \mathbf{Y b}-\Delta$ Psap4\#5 & 10.94 & 76.71 & 100 & $70.1 \%$ \\
${ }^{\mathbf{1 7 6}} \mathbf{Y b}-\mathbf{E P 1 5 8 8 Y}$ & 50 & 302.0 & 100 & $60.4 \%$ \\
\hline
\end{tabular}

A

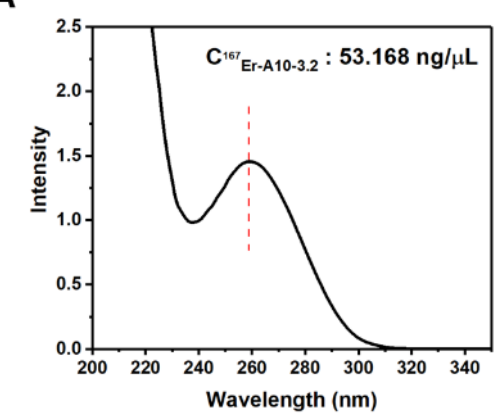

C

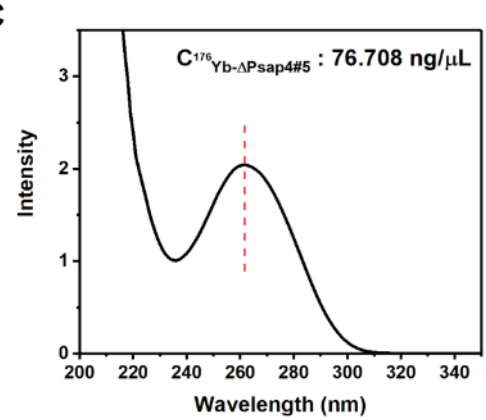

B

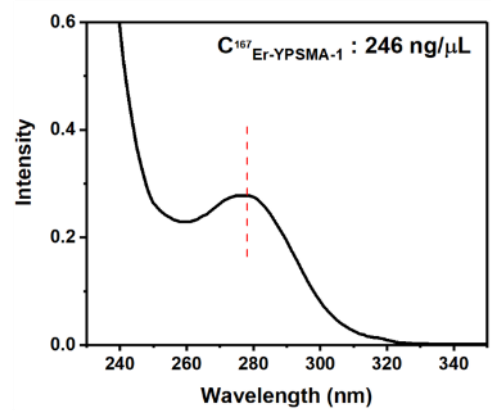

D

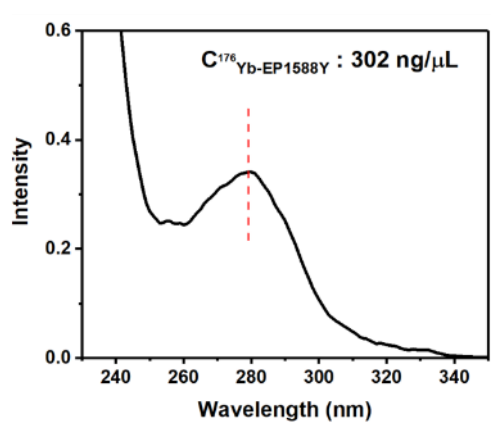

Figure S1 The characteristic UV absorption peak and the concentration of the mass tags investigated by Nano-100 Micro-Spectrophotometer: UV spectra of(A) ${ }^{167} \operatorname{Er}-\mathrm{A} 10$ 3.2 (B) ${ }^{167}$ Er-YPSMA-1 (C) ${ }^{176}$ Yb- $\Delta$ Psap4\#5 (D) ${ }^{176}$ Yb-EP1588Y. 
A

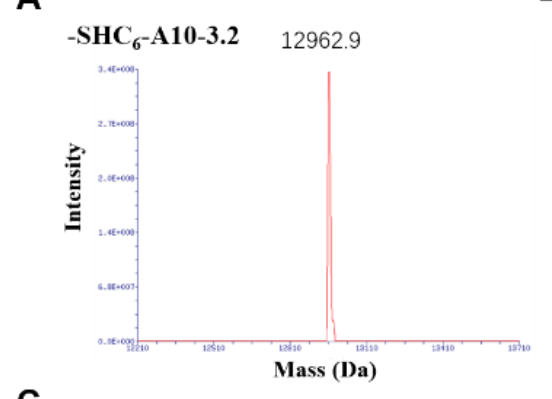

C

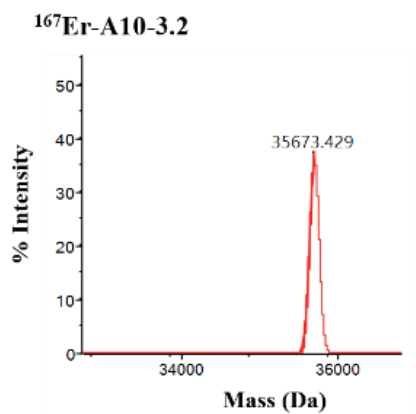

B

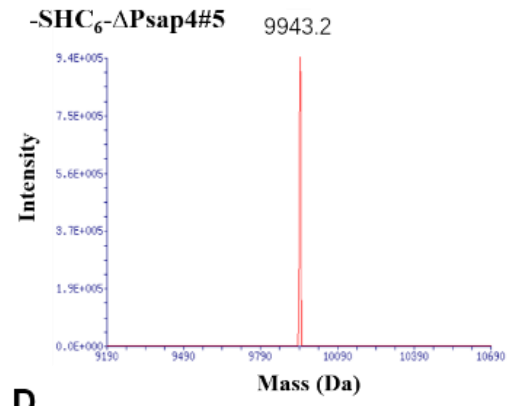

D

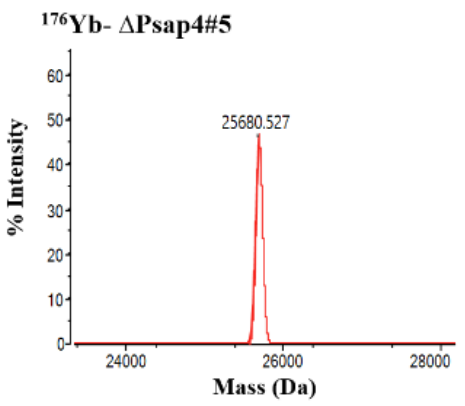

Figure S2 The mass spectrum of naked aptamers and metal-labeled aptamers. (A-B) the molecular weight of $-\mathrm{SHC}_{6}-\mathrm{A} 10-3.2$ and $-\mathrm{SHC}_{6}-\Delta \mathrm{Psap} 4 \# 5$ investigated by Thermo LCQ Deca XP plus Mass Spectrometer. (C-D) the molecular weight of ${ }^{167} \mathrm{Er}-\mathrm{A} 10-3.2$ and ${ }^{176} \mathrm{Yb}-\Delta \mathrm{Psap} 4 \# 5$ investigated by MALDI-TOF mass spectrometry
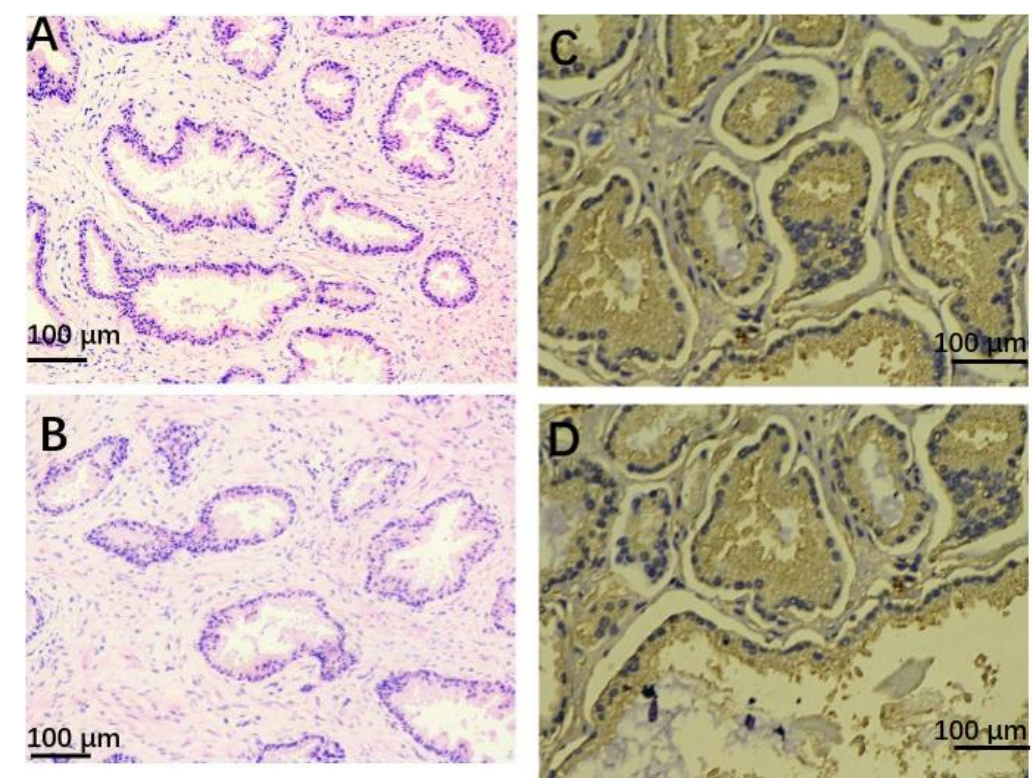

Figure S3 (A-B) Representative photomicrographs obtained from hematoxylin and eosin (H\&E) stained $\mathrm{PaC}$ tissue section. (C-D) Corresponding immunohistochemical stains for PSMA expression. Epitope retrieval temperature: $96^{\circ} \mathrm{C}$. 

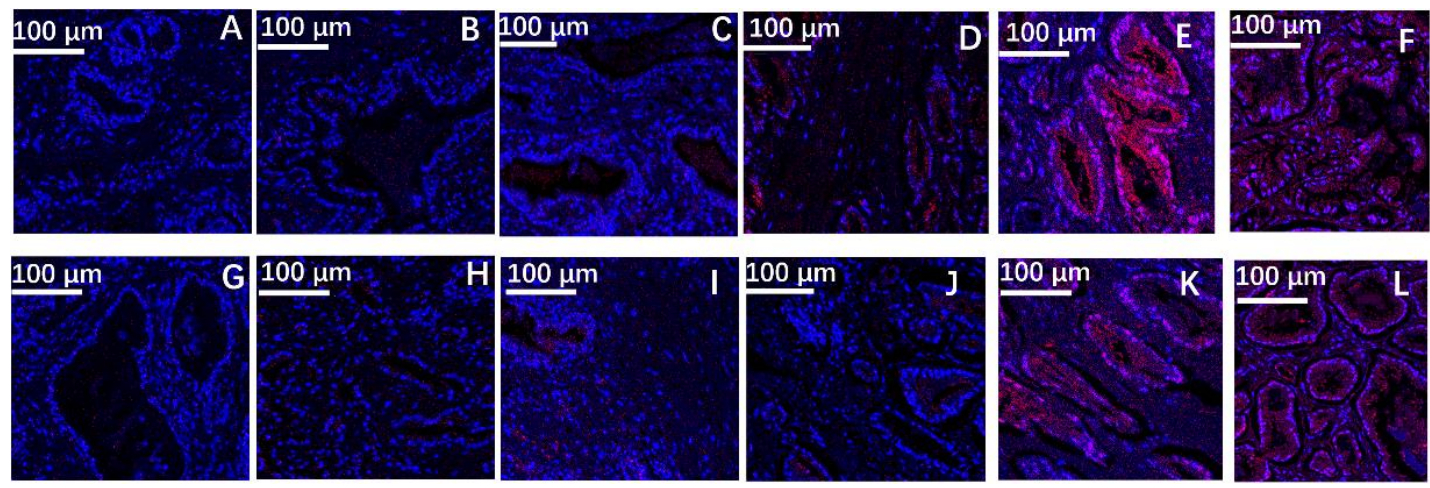

Figure S4 IMC images of Paraffin embedded $\mathrm{PaC}$ tissue section: probed by ${ }^{167} \mathrm{Er}-$ YPSMA-1 at concentration of (A) $1 \mu \mathrm{g} / \mathrm{mL}$, (B) $2 \mu \mathrm{g} / \mathrm{mL},(C) 3 \mu \mathrm{g} / \mathrm{mL}$, (D) $4 \mu \mathrm{g} / \mathrm{mL},(\mathrm{E})$ $5 \mu \mathrm{g} / \mathrm{mL}$ and (F) $10 \mu \mathrm{g} / \mathrm{mL}$; probed by ${ }^{167} \mathrm{Er}-\mathrm{A} 10-3.2$ at concentration of $(\mathrm{G}) 10 \mathrm{nM}$, (H)25 nM, (I)50 nM, (J) $75 \mathrm{nM},(\mathrm{K}) 100 \mathrm{nM}$ and (L)200 nM. Epitope retrieval temperature: $96^{\circ} \mathrm{C}$.
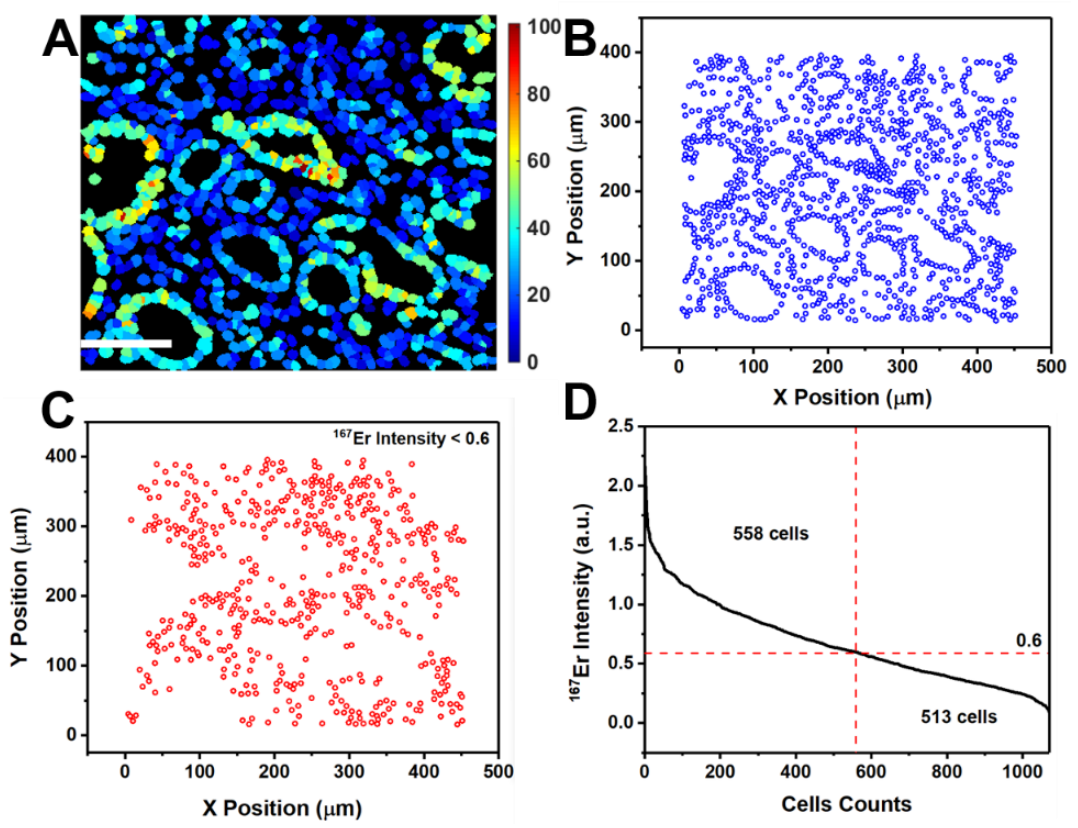

Figure S5 The percentage of overexpressed PSMA of the PaC tissue section stained by ${ }^{167} \mathrm{Er}-\mathrm{YPSMA}-1$ under $96{ }^{\circ} \mathrm{C}$ epitope retrieval is $52.1 \%$. (A) the heatmap image of ${ }^{167} \mathrm{Er}$ channel, scale bar:100 $\mu \mathrm{m}$. (B) the position coordinates of all types of cells of $\mathrm{PaC}$ tissue section. (C) the position coordinates of the cells in which the intensity of ${ }^{167} \mathrm{Er}$ signal is lower than 0.6. (D) the relationship of the intensity of ${ }^{167} \mathrm{Er}$ channel and the whole cells. 

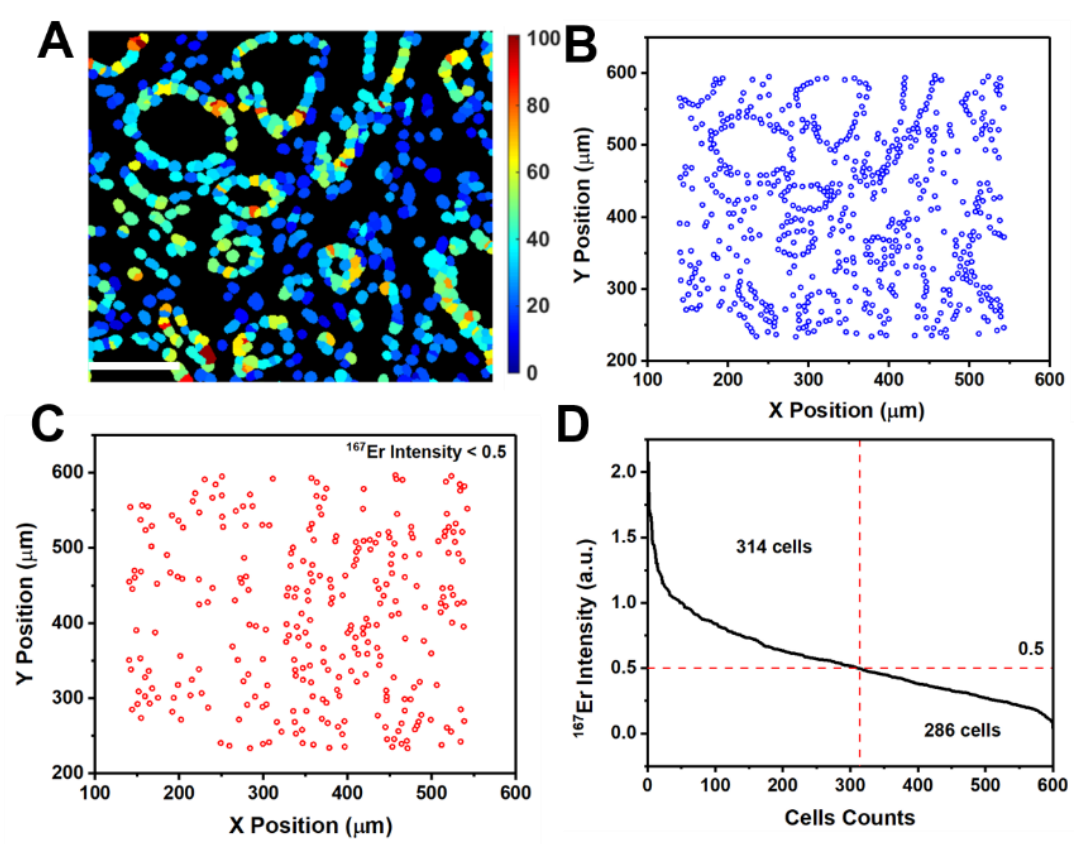

Figure S6 The percentage of overexpressed PSMA of the PaC tissue section stained by ${ }^{167} \mathrm{Er}-\mathrm{A} 10-3.2$ under $96{ }^{\circ} \mathrm{C}$ epitope retrieval is $52.3 \%$. (A) the heatmap image of ${ }^{167} \mathrm{Er}$ channel, scale bar:100 $\mu \mathrm{m}$. (B) the position coordinates of all types of cells of PaC tissue section. (C) the position coordinates of the cells in which the intensity of ${ }^{167} \mathrm{Er}$ signal is lower than 0.5 . (D) the relationship of the intensity of ${ }^{167} \mathrm{Er}$ channel and the whole cells.
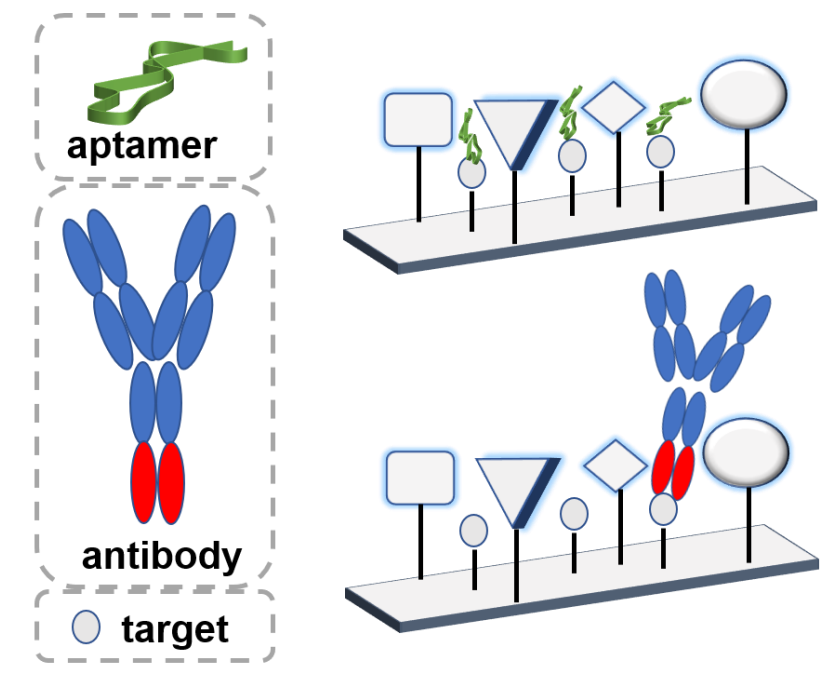

Figure S7 Scheme describing the hypothesis that the smaller size of the aptamers would allow a higher labeling density than antibodies. The larger size of the antibodies prevents them from reaching every epitope. 


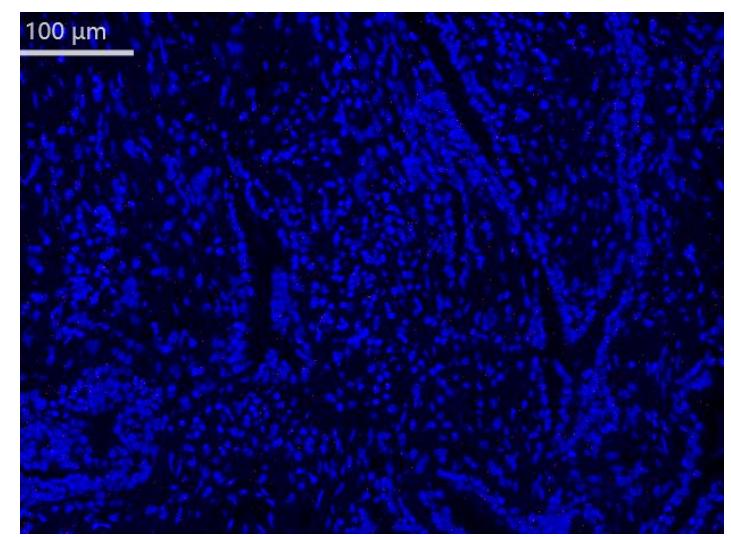

Figure S8 Blocking Experiment. The IMC image of the PaC section tissue (Red for PSMA, blue for DNA) stained by A10-3.2 and ${ }^{167}$ Er-A10-3.2, successively. Epitope retrieval temperature: $96^{\circ} \mathrm{C}$.

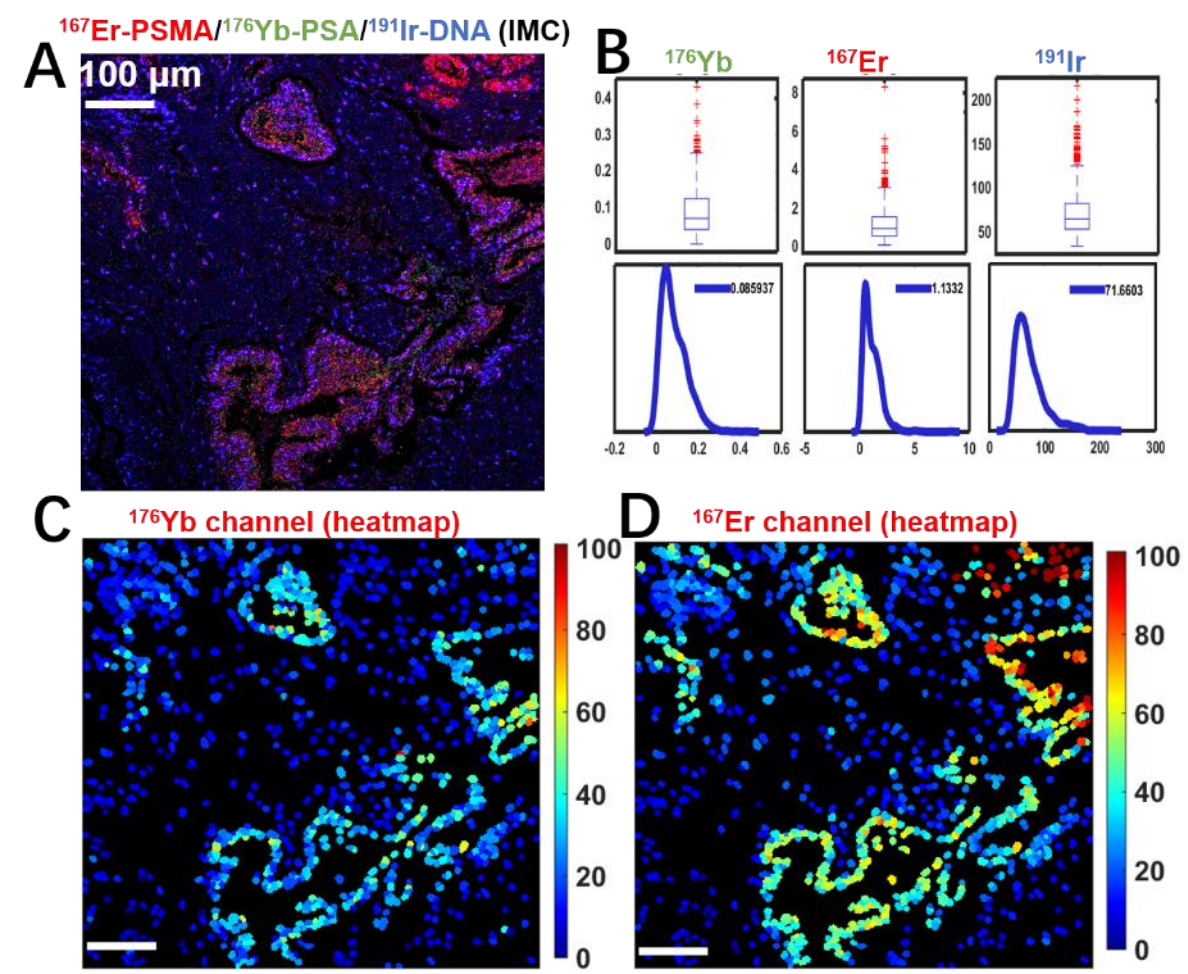

Figure S9 Panel: PSMA, PSA. IMC images of PaC tissue section (A) probed by ${ }^{167} \mathrm{Er}-$ YPSMA-1 and ${ }^{176}$ Yb-EP1588Y antibodies (Red for PSMA, green for PSA, blue for DNA) and (B) corresponding signal intensity of ${ }^{176} \mathrm{Yb},{ }^{167} \mathrm{Er}$ and ${ }^{191} \mathrm{Ir}$ channels. (C-D) heatmap images of ${ }^{176} \mathrm{Yb}$ and ${ }^{167} \mathrm{Er}$ channels indicating the distribution of PSMA and PSA of the PaC tissue, Epitope retrieval temperature: $96{ }^{\circ} \mathrm{C}$, Scale bar: $100 \mu \mathrm{m}$. 


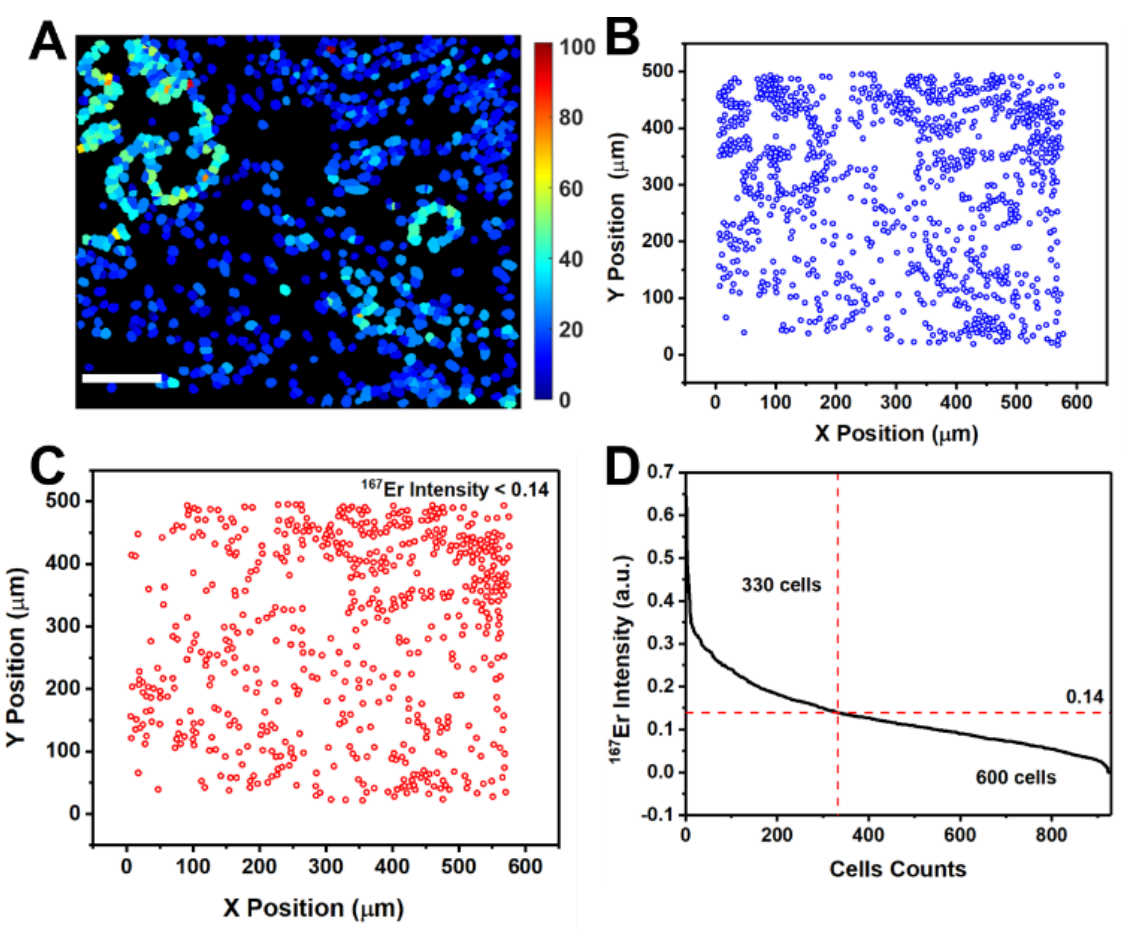

Figure S10 The percentage of overexpressed PSMA of the PaC tissue section stained by ${ }^{167} \mathrm{Er}-\mathrm{A} 10-3.2$ under $96{ }^{\circ} \mathrm{C}$ epitope retrieval is $35.5 \%$. (A) the heatmap image of ${ }^{167} \mathrm{Er}$ channel, scale bar: $100 \mu \mathrm{m}$ (B) the position coordinates of all types of cells of $\mathrm{PaC}$ tissue section. (C) the position coordinates of the cells in which the intensity of ${ }^{167} \mathrm{Er}$ signal is lower than 0.14. (D) the relationship of the intensity of ${ }^{167} \mathrm{Er}$ channel and the whole cells. 

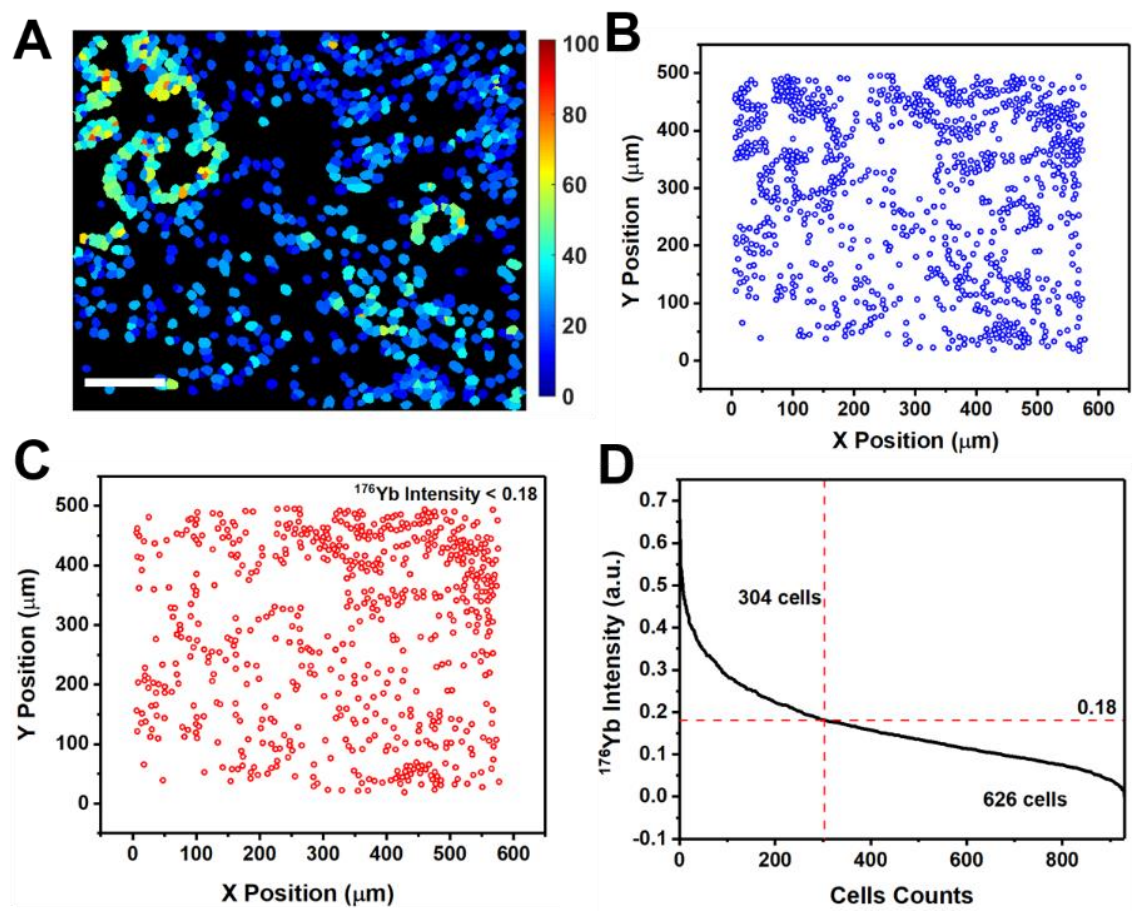

Figure S11 The percentage of overexpressed PSA of the PaC tissue section stained by ${ }^{167} \mathrm{Er}-\mathrm{A} 10-3.2,{ }^{176} \mathrm{Yb}-\triangle$ Psap4\#5 under $96{ }^{\circ} \mathrm{C}$ epitope retrieval is $32.7 \%$. (A) the heatmap image of ${ }^{167} \mathrm{Er}$ channel, scale bar:100 $\mu \mathrm{m}$ (B) the position coordinates of all types of cells of $\mathrm{PaC}$ tissue section. (C) the position coordinates of the cells in which the intensity of ${ }^{176} \mathrm{Yb}$ signal is lower than 0.18 . (D) the relationship of the intensity of ${ }^{176} \mathrm{Yb}$ channel and the whole cells. 


\section{Experiment section}

\section{Metal-chelating polymer}

1. Spin polymer tube for 10 seconds in a microfuge to ensure the reagent is at the bottom of the tube.

2. Resuspend polymer with $95 \mu \mathrm{L}$ of L-Buffer.

3. Mix thoroughly by pipetting.

4. Add $5 \mu \mathrm{L}$ of Lanthanide metal solution $\left({ }^{167} \mathrm{Er}\right.$ or ${ }^{176} \mathrm{Yb}$ ) to tube (final concentration: $2.5 \mathrm{mM})$.

5. Mix thoroughly by pipetting.

6. Incubate at $37^{\circ} \mathrm{C}$ for $30-40$ minutes.

7. Add $200 \mu \mathrm{L}$ of L-Buffer to a $3 \mathrm{kDa}$ filter.

8. Add the $100 \mu \mathrm{L}$ metal-loaded polymer mixture to the filter containing the $200 \mu \mathrm{L} \mathrm{L}$ Buffer to the wash.

9. Centrifuge at $12,000 \mathrm{x} \mathrm{g}$ for 25 minutes at RT.

10. Repeat the wash by adding $400 \mu \mathrm{L}$ of C-Buffer to the filter and centrifuge at $12,000 \mathrm{x}$ $\mathrm{g}$ for 30 minutes at RT.

11. Retrieve $3 \mathrm{kDa}$ spin filter containing the Lanthanide-loaded Polymer from the centrifuge and discard column flow through

12. Using a pipette, re-suspend the lanthanide-loaded polymer in $60 \mu \mathrm{L}$ of $\mathrm{C}$-Buffer (half for antibody conjugation, half for aptamer conjugation)

\section{Metal-labeled antibody}

The metal-labeled antibody is totally according to the protocol of Fludigum. The website is showed as below:

http://cn.fluidigm.com/binaries/content/documents/fluidigm/search/hippo\%3Aresultse t/maxpar-antibody-labeling-kit-pr-prd002/fluidigm\%3Afile

\section{Metal-labeled aptamer}

Details of synthesis and separation procedure for the metal-labeled aptamers:

Partially Reduce the thiol modified aptamer

1. $1 \mathrm{nmol}$ thiol modified aptamer was reduced by $10 \mathrm{mM}$ TCEP (final concentration) 
2. Incubate at room temperature for 30 minutes

\section{Purify the Partially Reduced aptamer}

3. Add $300 \mu \mathrm{L}$ of $\mathrm{C}$-Buffer to a $3 \mathrm{kDa}$ spin filter

4. Add the mixture of 2 to the spin filter containing the C-Buffer

5. Centrifuge $15,000 \times \mathrm{g}$ for 12 minutes at RT

6. Discard flow through

7. Add $400 \mu \mathrm{L}$ of $\mathrm{C}$-Buffer to the spin filter

8. Centrifuge $15,000 \times \mathrm{g}$ for 12 minutes at RT

\section{Conjugate aptamer with Lanthanide-loaded Polymer}

9. Transfer $30 \mu \mathrm{l}$ lanthanide-loaded polymer to the corresponding partially reduced aptamer in the $3 \mathrm{kDa}$ spin filter

10. Mix briefly by pipetting

11. Incubate at $37^{\circ} \mathrm{C}$ for 2 hours

12. 0.5 M TCEP stock was added to a final concentration of $5 \mathrm{mM}$

13. Incubate at $37^{\circ} \mathrm{C}$ for $30 \mathrm{~min}$ to reduce unconjugated oligonucleotides

14. Add $300 \mu \mathrm{L}$ DEPC-treated water

15. Mix briefly by pipetting

\section{Wash metal conjugated aptamer}

16. Transfer the mix into a $30 \mathrm{kDa}$ spin filter

17. Centrifuge $14,000 \times \mathrm{g}$ for 12 minutes

18. Discard flow through

19. Repeat 3 more times with $400 \mu \mathrm{L}$ of DEPC-treated water

\section{Recover Metal Conjugated aptamer}

20. Add $50 \mu \mathrm{L}$ of DEPC-treated water to the $30 \mathrm{kDa}$ spin filter, pipette to mix and rinse the walls of the filter

21. Invert the $30 \mathrm{kDa}$ spin filter over to a new collection tube

22. Centrifuge the inverted filter/collection tube assembly at $1200 \times \mathrm{g}$ for 2 minutes

23. Remove the inverted spin filter from the collection tube, rinse walls of the filter with an additional $50 \mu \mathrm{L}$ of DEPC-treated water and replace it, inverted, back to the collection tube 
24. Centrifuge the inverted filter/collection tube assembly at $1200 \times \mathrm{g}$ for 2 minutes Yield Determination and Storage of metal Conjugated aptamer

25. Quantify the conjugated aptamer by measuring the absorbance at $260 \mathrm{~nm}$ against a DEPC-treated water blank

26. Store at $4^{\circ} \mathrm{C}$ until ready to titrate and IMC experiment 